
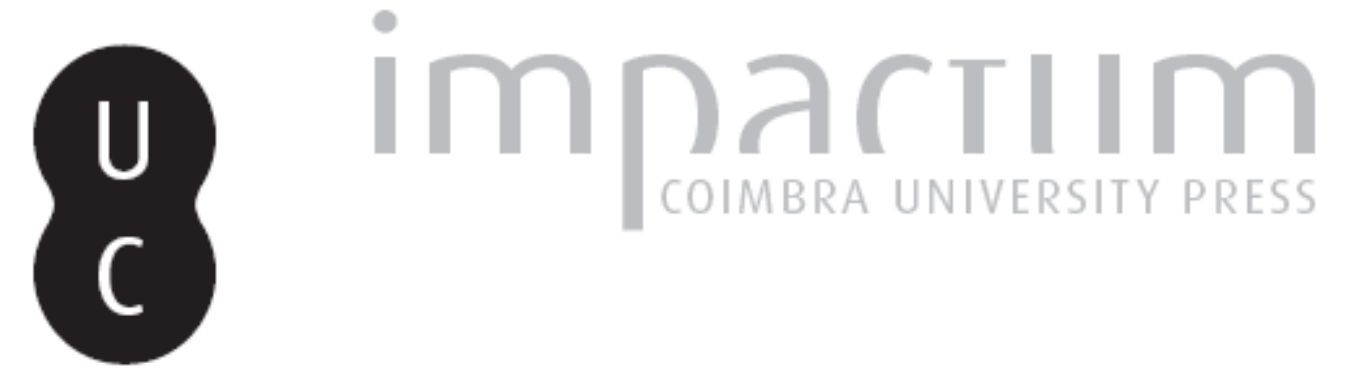

\title{
[Recensão a] LIBERO ARBITRIO. STORIA DI UNA CONTROVERSIA FILOSOFICA
}

\author{
Autor(es): Mazzetti, Manuel
}

Publicado por: Annablume Clássica; Imprensa da Universidade de Coimbra

URL persistente:

URl:http://hdl.handle.net/10316.2/37018

DOI:

DOI:http://dx.doi.org/10.14195/1984-249X_15_17

Accessed : $\quad$ 26-Apr-2023 03:06:23

A navegação consulta e descarregamento dos títulos inseridos nas Bibliotecas Digitais UC Digitalis, UC Pombalina e UC Impactum, pressupõem a aceitação plena e sem reservas dos Termos e Condições de Uso destas Bibliotecas Digitais, disponíveis em https://digitalis.uc.pt/pt-pt/termos.

Conforme exposto nos referidos Termos e Condições de Uso, o descarregamento de títulos de acesso restrito requer uma licença válida de autorização devendo o utilizador aceder ao(s) documento(s) a partir de um endereço de IP da instituição detentora da supramencionada licença.

Ao utilizador é apenas permitido o descarregamento para uso pessoal, pelo que o emprego do(s) título(s) descarregado(s) para outro fim, designadamente comercial, carece de autorização do respetivo autor ou editor da obra.

Na medida em que todas as obras da UC Digitalis se encontram protegidas pelo Código do Direito de Autor e Direitos Conexos e demais legislação aplicável, toda a cópia, parcial ou total, deste documento, nos casos em que é legalmente admitida, deverá conter ou fazer-se acompanhar por este aviso.

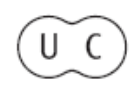




\section{5}

jul/dez

2015

issn 2179-4960

e-issn 1984-249-X
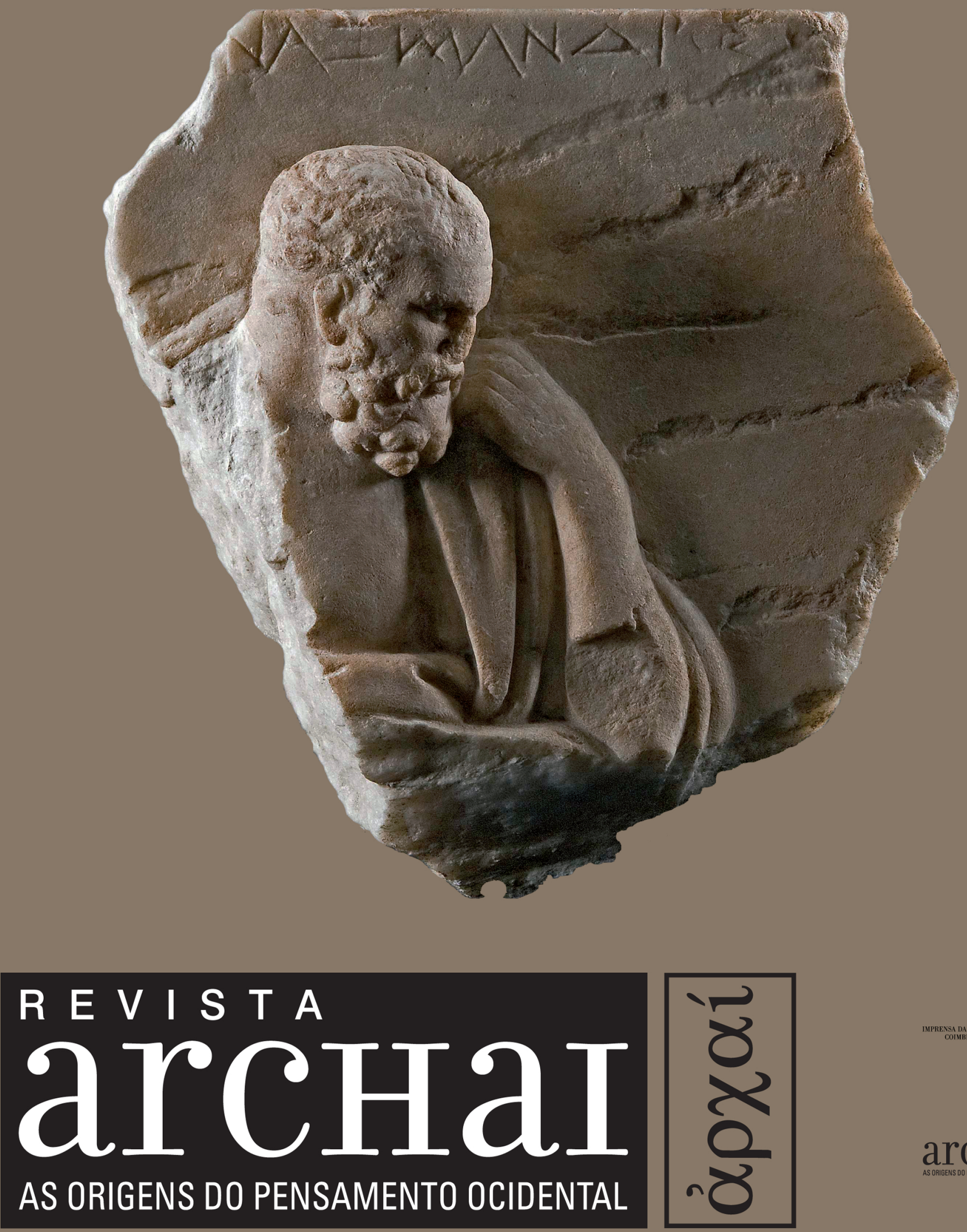

ARCHAI JOURNAL: ON THE ORIGINS OF WESTERN THOUGHT

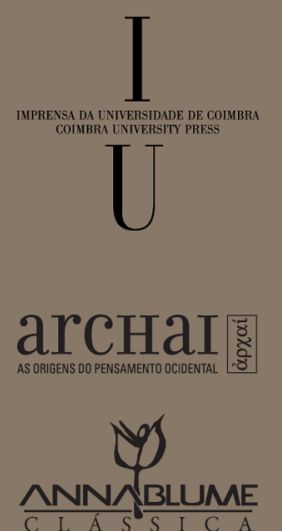




\section{LIBERO ARBITRIO. STORIA DI UNA CONTROVERSIA FILOSOFICA}

MAZZETTI, M. (2015). Resenha. DE CARO, M.; MORI, M.; SPINELLI, E. EDS. (2014). Libero arbitrio. Storia di una controversia filosofica. Roma, Carocci.

Archai, n. 15, jul. - dez., p. 165-167

D0I: http://dx.doi.org/10.14195/1984-249X_15_17
* La Sapienza University of Rome - Roma, Itália manmazze88@gmail.com

\section{Manuel Mazzetti}

Il problema del libero arbitrio ha ricevuto un'attenzione costante durante la storia della filosofia, dall'antichità fino ai nostri giorni. Il volume curato da De Caro, Mori e Spinelli offre una panoramica esauriente sulle modalità con cui il tema è stato affrontato nell'intera storia della filosofia. Con ciò non si deve intendere che vi sia stato, in un arco temporale che supera i due millenni, un solo problema del libero arbitrio: come premettono i curatori, la peculiarità di questo tema rispetto ad altre questioni che solcano l'intero cammino della storia della filosofia è quella di aver ricevuto non soltanto una miriade di diverse risposte, ma anche una serie di formulazioni diverse: libero arbitrio in relazione ora alla volontà, ora all'azione; avversario del determinismo o compatibile con esso; minacciato da fattori fisici (le leggi naturali; il fato), teologici (la provvidenza), logici (il problema della verità delle affermazioni sul futuro); e così via. L'esigenza ermeneutica di cogliere il problema nella formulazione specifica di ogni epoca storica in cui viene affrontato è tanto più cogente quanto più si risale indietro nel tempo: i capitoli riguardanti la filosofia antica, che considereremo in questa sede, si impegnano pertanto a fornire una ricostruzione, per quanto possibile, fedele ai testi in nostro possesso, senza proiettarvi illecitamente schemi mentali tipici della contemporaneità. 
Nel primo capitolo Franco Trabattoni ripercorre la teoria platonica della libertà umana - che trova la sua più celebre e felice esposizione nel mito di Er della Repubblica - considerata come antitetica sia alla visione «pessimistica» dell'epica e degli autori tragici, secondo la quale l'uomo, per quanto si sforzi di impiegare al meglio la sua razionalità per perseguire la virtù, resta in ultima analisi soggiogato alla volontà arbitraria e insondabile degli dèi, che possono manovrarlo e ingannarlo a loro discrezione; sia alla tesi, di matrice soprattutto eraclitea, per cui l'agire umano, anche quando si affranchi dall'influenza degli dèi, resta ugualmente condizionato tanto dalla necessità interna costituita dal suo carattere e dai suoi desideri, quanto dai processi naturali, in cui le cicliche trasformazioni degli elementi sono regolate dal destino. Com'è noto, Platone risponde che ciascuna anima, prima della nascita, ha avuto la possibilità di scegliere il proprio demone, e che pertanto la responsabilità delle azioni che da esso conseguono non è della divinità, bensì dell'uomo stesso. Seppur libera, la scelta prenatale sembra tuttavia condizionare in toto le future azioni di chi l'ha compiuta, e ciò contrasta con l'intento pedagogico di plasmare e modificare il carattere, perseguito in altri luoghi della Repubblica stessa. Trabattoni osserva che «formalmente parlando» (p. 19) questo problema risulta «inaggirabile», ma insiste anche sulla necessità di stemperare l'apparente gravità di tale incoerenza tenendo presente il carattere metaforico e polemico dell'intero mito di Er: in quanto mito, esso è una rappresentazione approssimativa della realtà a cui si riferisce. Nella parte finale del capitolo, l'autore si impegna a ricostruire in cosa consista, di fatto, la libertà difesa negli scritti platonici: premesso che il fine della vita umana non può essere scelto, in quanto consiste per natura nel raggiungimento della felicità, la libertà deve riguardare la scelta dei mezzi adeguati a perseguire quel fine. La conseguenza, solo apparentemente paradossale, di questa tesi è che la libertà non consiste affatto nella possibilità di scegliere in modo arbitrario e indiscriminato ciò che si vuole: se, per esempio, al piccolo Liside del dialogo omonimo non venisse proibito di guidare il carro del padre, ed egli fosse perciò del tutto libero di farlo, ciò non contribuirebbe affatto alla sua feli- cità, bensì alla sua rovina. In tal senso, una libertà totale riguardo ai mezzi coincide con l'ignoranza: l'esito di questa riflessione sembra essere, pertanto, che minore è la libertà riguardo ai mezzi, ovvero le alternative fra cui è auspicabile scegliere, maggiore è la libertà di poter ottenere il fine.

Nel secondo capitolo Carlo Natali raccoglie innanzitutto $\mathrm{i}$ passi delle opere di Aristotele più significativi per ricostruire il suo pensiero sul libero arbitrio: la lettura diretta dei testi è, qui più che altrove, necessaria per offrire un quadro obiettivo della posizione aristotelica, poiché essa è stata oggetto, dall'antichità fino ad oggi, di interpretazioni disparate e spesso fra di loro contraddittorie. È noto infatti che gli scritti dello Stagirita enfatizzano in molti luoghi la contingenza di tutto ciò che riguarda il mondo sublunare (che può essere o non essere, e in cui gli eventi si verificano «per lo più», ma ammettono eccezioni), e la responsabilità dell'uomo per una classe di azioni che dipendono da lui, cioè non sono forzate da agenti esterni. I Peripatetici antichi interpretarono queste asserzioni in senso indeterministico, ma sono possibili altresì letture di stampo deterministico, e non si può neppure escludere che Aristotele non si fosse affatto posto il problema. I due punti problematici su cui si è incentrato il dibattito contemporaneo, messi in luce da Natali, sono la vaghezza del concetto di contingenza - che sembra riguardare più casi generici (l'acqua può bollire o meno), che le loro singole istanziazioni (in certe circostanze, per es. in presenza di una fonte di calore, l'acqua bolle necessariamente) - e soprattutto le ambiguità relative alla formazione del carattere: un agente è responsabile delle azioni che discendono dal suo carattere perché a lui è imputabile la formazione del carattere stesso; ma tale formazione è influenzata a sua volta da fattori esterni e culturali, e in ultima analisi non sembra perciò libera. Natali inclina per un'interpretazione cautamente indeterminista e conclude che Aristotele, pur non avendo impostato il problema in maniera del tutto rigorosa, rappresenta un momento importante nel dibattito sui rapporti fra libero arbitrio e determinismo.

In età ellenistica la questione assume un'importanza dirompente, benché - come osservano Emidio Spinelli e Francesco Verde, autori del capitolo sulle scuole ellenistiche - $\mathrm{i}$ termini in cui essa viene 
affrontata non debbano essere anacronisticamente sovrapposti a quelli del dibattito attuale, con cui pure presentano innegabili analogie. Il merito principale della sezione su Epicuro è quello di riportare l'attenzione sui frammenti del XXV libro dell'opera Sulla natura, oltre che sui più celebri passi in cui viene riferita la dottrina del clinamen: secondo Verde, quest'ultima sarebbe stata introdotta in una fase successiva alla redazione del XXV libro in cui infatti, stando almeno alle parti superstiti, non trova menzione alcuna. Nell'opera Sulla natura Epicuro avrebbe difeso la capacità della mente di potersi determinare autonomamente, nonostante la necessità dei moti degli atomi, non essendo totalmente soggetta ai condizionamenti esterni e a quelli della sua stessa struttura atomica. Con la successiva introduzione del clinamen, ovvero della possibilità degli atomi di deviare dalla propria traiettoria in un momento indeterminato spazio-temporalmente, Epicuro avrebbe offerto una spiegazione in termini fisici di quell'autonomia già precedentemente teorizzata. La seconda sezione del capitolo ripercorre sinteticamente i punti chiave della teoria degli Stoici, che costituiscono i deterministi par excellence dell'antichità. I presupposti che inducono ad ammettere che da ogni causa consegua inevitabilmente un solo effetto, e che il mondo costituisca una sorta di «rete» in cui infinite cause si intrecciano secondo modalità per lo più ignote all'osservatore umano, sono tuttavia di ordine metafisico ancor prima che scientifico: il cosmo è infatti, secondo gli Stoici, un intero le cui parti sono tutte correlate fra loro secondo un criterio razionale, sancito da un principio divino immanente al cosmo stesso. Spinelli ripercorre sinteticamente il diverso approccio con cui Cleante, Crisippo ed Epitteto si impegnarono a mostrare la compatibilità tra la loro fisica rigorosamente deterministica e, in campo etico, la responsabilità umana: al di là delle differenti formulazioni, la tesi comune è che le azioni provenienti dall'iniziativa umana, ancorché determinate in maniera univoca, siano tuttavia imputabili al soggetto agente, per il fatto stesso di provenire da facoltà a lui interne, e non da costrizioni esterne.

L'anello di congiunzione fra il terzo e il quarto capitolo è costituito da Carneade, di cui tuttavia vengono proposte due differenti letture: Spinelli e
Verde insistono sugli aspetti scettici del pensiero di Carneade, ovvero sulla natura puramente dialettica delle sue critiche, vòlte a mostrare che ciascuna delle tesi contrastanti difese dalle scuole filosofiche è parimenti insoddisfacente; Trabattoni si appella invece all'appartenenza di Carneade all'Accademia per scorgere, dietro alle sue critiche, l'affermazione di una dottrina positiva di matrice platonica. La parte rimanente del quarto capitolo, curato sempre da Trabattoni, e l'intero capitolo successivo, curato ancora da Natali, sono dedicate a ricostruire le reazioni delle scuole dogmatiche - rispettivamente, l'Accademia e il Peripato - al determinismo stoico, tese a ritagliare uno spazio alla libertà dell'agire umano più ampio della mera provenienza da principî interni. Il cosiddetto «medio-platonismo» elaborò, sulla base del mito di Er, la dottrina del fato come legge condizionale, esprimibile nella forma: «se sceglierai una certa cosa, deriveranno certe conseguenze»; la scelta posta dall'apodosi è in nostro potere, e non è determinata dal fato, a cui sono imputabili invece gli esiti inevitabili che da essa scaturiscono. In Plotino e negli altri neoplatonici, invece, il problema del libero arbitrio è inquadrato nella prospettiva più schiettamente teologica del provvidenzialismo: la divinità agisce per il meglio e determina il ruolo che ciascun individuo riveste nel mondo; ma solo a l'individuo spetta il compito di recitare bene o male, come un buon o cattivo attore, la parte che la provvidenza gli ha assegnato. Infine, il Peripato, e in particolare Alessandro di Afrodisia, equiparava il fato alla natura individuale degli enti sublunari, ovvero a quel complesso di caratteristiche che, pur condizionando gli eventi con una certa regolarità, ammettono eccezioni.

In conclusione, il libro sul libero arbitrio, di cui abbiamo velocemente ripercorso le sezioni riguardanti la filosofia antica, ha il merito di fornire un quadro generale dell'approccio di ciascun autore o scuola filosofica alla questione, e nel contempo di dare una visione essenziale, ma precisa e aggiornata alla bibliografia più recente, degli aspetti problematici e delle più interessanti interpretazioni moderne.

Submetido em Fevereiro de 2015 e aprovado em Março de 2015. 